\title{
MicroRNA-29a-3p enhances dental implant osseointegration of hyperlipidemic rats via suppressing dishevelled 2 and frizzled 4
}

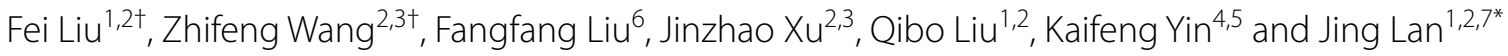

\begin{abstract}
Background: Fine osseointegration is the basis of long-term survival of implant. In our previous study, we observed a strong correlation between hyperlipidemia and compromised osseointegration. MicroRNA-29a-3p (miR-29a-3p) has been discovered to participate in bone marrow mesenchymal stem cells (BMSCs) differentiation. However, the role and the underlying mechanisms of hyperlipidemia and miR-29a-3p in osseointegration still remain obscure.
\end{abstract}

Results: In peri-implant bone tissues of hyperlipidemia rats, bone mass, mineralization and bone trabecula formation were weakened. Alkaline phosphatase (ALP) and runt-related transcription factor 2 (Runx2), and miR-29a-3p expression were reduced. While in normal rats, implant-bone interfaces were filled with dense new bone and ALP, Runx2 and miR-29a-3p were up-regulated. Overexpressed miR-29a-3p can reverse the adverse effect of hyperlipidemia on osseointegration. Implants were tightly integrated with the surrounding dense new bone tissues, and ALP as well as Runx2 mRNAs were enhanced in miR-29a-3p overexpressed and hyperlipidemia rats, while little peri-implant bone tissue existed, ALP and Runx2 deregulated on miR-29a-3p inhibited rats. Dishevelled 2 (Dvl2) mRNA was declined in peri-implant bone tissue of high-fat (HF) group than normal group, while frizzled 4 (Fzd4) mRNA declined on day 5 and increased from day 10 to day 20 after implantation in hyperlipidemia rats than in normal rats. Next, BMSCs were cultured under HF or normal medium in vitro. In the HF group, ALP activity and mineralization, ALP and Runx2 mRNAs and proteins expression, and miR-29a-3p expression were suppressed, while adipogenesis was increased, as a result, cytoskeletons were sparse and disordered compared to control group. However, when miR-29a-3p was overexpressed in BMSCs, ALP activity, ALP, Runx2, Dvl2 and Fzd4 mRNAs and proteins expressions were up-regulated. As miR-29a-3p was inhibited in BMSCs, the reverse results were obtained. In addition, promoter assay revealed that miR-29a-3p can directly suppress Wnt/ $\beta$-catenin pathway related Dvl2 and Fzd4 through binding to their 3'-UTR.

Conclusions: MiR-29a-3p facilitated implant osseointegration via targeting Wnt/ $\beta$-catenin pathway-related DvI2 and Fzd4. MiR-29a-3p/Dvl2/Fzd4 may serve as a promising therapeutic target for hyperlipidemia osseointegration.

Keywords: miR-29a-3p, Hyperlipidemia, Dvl2, Fzd4, Osseointegration

\footnotetext{
*Correspondence: lanjing@sdu.edu.cn

${ }^{\dagger}$ Fei Liu and Zhifeng Wang are co-first authors with equal contributions

to the article

${ }^{7}$ Department of Prosthodontics, School of Dentistry, Shandong

University, Jinan, China

Full list of author information is available at the end of the article
}

(c) The Author(s) 2018. This article is distributed under the terms of the Creative Commons Attribution 4.0 International License (http://creativecommons.org/licenses/by/4.0/), which permits unrestricted use, distribution, and reproduction in any medium, provided you give appropriate credit to the original author(s) and the source, provide a link to the Creative Commons license, and indicate if changes were made. The Creative Commons Public Domain Dedication waiver (http://creativecommons.org/ publicdomain/zero/1.0/) applies to the data made available in this article, unless otherwise stated. 


\section{Background}

High-fat diet (HFD) can induce hyperlipidemia, which was characterized by an elevation of lipids in the bloodstream. Hyperlipidemia significantly damaged bone metabolism, decreased bone mineral density, volume and strength, and increased the risk of bone fracture and osteoporosis [1-3]. Excellent osseointegration was associated with long-term success rate of implants. However, hyperlipidemia leaded to increased implant loss, and reduced strength and bone formation of implant-bone interface [3-5].

As the incidence of hyperlipidemia has been increasing yearly [6], it is significant to study the underlying molecular mechanism of hyperlipidemia on implant osseointegration. Bone marrow mesenchymal stem cells (BMSCs), known for its pluripotent differentiation capacity, were the progenitor cells for osteoblasts, and played a substantial role in bone formation and regeneration $[7,8]$. Xu et al. [9] demonstrated that BMSCs transplantation significantly enhanced new bone formation, but hyperlipidemia compromised homing efficiency of systematically transplanted BMSCs and inhibited bone regeneration. Alkaline phosphatase (ALP), engaged in bone matrix mineralization, and runt-related transcription factor 2 (Runx2), a master transcription factor of osteogenesis, were considered as early marker genes of osteogenesis [10-17].

A great amount of microRNAs (miRNAs) has been shown to regulate bone formation and regeneration as well as implant osseointegration [18, 19]. Many miRNAs were aberrantly expressed during osteogenesis in hyperlipidemia models, including miR-29b, miR-17, miR-146, miRNA-23, miR-34c. [20-24]. Interestingly, miR-29a-3p has been confirmed as a positive regulator on osteogenesis [25-27], and was increased during osteogenic differentiation in mice [27], but its role in osseointegration is not clear.

Wnt/ $\beta$-catenin pathway played significant roles in osteogenic differentiation of BMSC [28-30]. Osteogenesis was enhanced by the activation of Wnt/ $\beta$-catenin pathway [31]. Accordingly, it is important to search for Wnt/ $\beta$-catenin signaling-related downstream genes of miR-29a-3p to understand the underlying mechanism of miR-29a-3p on osseointegration.

Based on previous studies [32, 33], we have successfully established hyperlipidemia rat models by inserting implants into the bilateral femoral metaphysis, a method widely adopted by other researches [33-35]. To further elucidate the detailed function of miR-29a-3p during BMSCs osteogenesis and the effect of hyperlipidemia on miR-29a-3p, relevant biological and functional assays were performed in vitro. Furthermore, online softwares were used to screen the $\mathrm{Wnt} / \beta$-catenin pathway mediated downstream target genes of miR-29a-3p. Dishevelled 2 (Dvl2), a cytoplasmic molecule of the Wnt/ $\beta$-catenin signaling [36, 37], and frizzled 4 (FZD4), an essential receptor of the $\mathrm{Wnt} / \beta$-catenin signal pathway $[38,39]$, were selected and validated as downstream targets of miR-29a-3p by dual luciferase reporter assays.

\section{Methods \\ Biological and functional experiments in vivo Animal preparation}

All Wistar rats (male, 6 weeks old and 180-220 g weigh), were purchased from the Experimental Animal Center of Shandong University (Ji'nan, China). All rats were housed in sterilized, pathogen-free, temperature controlled facility on a normal 12-h light/dark cycle, and standard diet and water were provided ad libitum.

Rats were randomly divided into normal and HF group $(\mathrm{n}=16$ in each group). HF group was fed on HFD (78.8\% basic feed, $10 \%$ lard, $10 \%$ egg yolk powder, $1 \%$ cholesterol, $0.2 \%$ bile salt), while normal group fed on normal diet. After 8 weeks, fasting blood samples were taken from the endocanthion vein of animals to determine serum lipid levels including low density lipoprotein (LDL), high density lipoprotein (HDL), triglyceride (TG) and total cholesterol (TC) using an autoanalyzer (Hitachi, Japan).

\section{Implant placement and tissue preparation}

General anesthesia was obtained by intraperitoneal injection of $10 \%$ chloral hydrate $(10 \mathrm{ml} / \mathrm{kg}$, Qilu Hospital, Jinan, China). For implant insertion, a hole $(1.3 \mathrm{~mm}$ diameter) was drilled on the site $5 \mathrm{~mm}$ away from the bilateral distal femoral metaphysis. Subsequently, the titanium implant (Shuangyang, China), rodshaped custom-made, $1.5 \mathrm{~mm}$ diameter and $2.5 \mathrm{~mm}$ length was inserted into the hole [33-35]. After operation, all rats received intramuscularly injection of penicillin $(0.06 \mathrm{ml} /$ $\mathrm{kg}$, once a day) for 3 postoperative days. 5, 10, 15, 20 days after implantation, 4 rats in each group were sacrificed by deep anesthesia with chloral hydrate. $1 \mathrm{~mm}$ bones around implants were dissected without surrounding muscles. All samples were frozen in liquid nitrogen rapidly.

\section{Hard tissue slices preparation and hematoxylin-eosin (HE) staining}

The samples, 10 days after implantation, were prepared for hard tissue slices. After fixation with $4 \%$ paraformaldehyde, the bone tissue with implant were embedded with resin, and sectioned in longitudinal direction parallel to the long axis of the implant by section cutter. About $30 \mu \mathrm{m}$ thick sections were obtained by grinding machine (E400CS, EXAKT Vertriebs Gmbh, Germany) and stained with hematoxylin-eosin (HE) by staining parallel adhesive tablet device (E401/402, EXAKT 
Vertriebs Gmbh, Germany). Implant-bone interface were observed by microscopy (Olympus, Japan).

\section{RNA extraction and quantitative reverse-transcription polymerase chain reaction( $q R T-P C R$ )}

5, 10, 15, 20 days after insertion, $1 \mathrm{~mm}$ femur tissues around implants were prepared for qRT-PCR. Total RNA of these bone tissues was isolated using Trizol reagent (TaKaRa, China) according to the manufacture's instruction. The first-strand cDNA was synthesized from $1 \mu \mathrm{g}$ of total RNA using PrimeScript ${ }^{\mathrm{TM}}$ II 1 st Strand cDNA Synthesis Kit (Takara, Dalian, China). Quantitative reverse-transcription polymerase chain reaction (qRT-PCR) was conducted with a standard SYBR Green PCR kit (TaKaRa, China) on the LightCycler 4801196 (Roche, Switzerland). The relative expressions of miR-29a-3p, and mRNA level expression of ALP, Runx2 were measured using the $2^{-\Delta \Delta C t}$ methods with GAPDH as an internal control. Primer sequences are listed in Table 1.

\section{Lentiviral vectors construction, miR-29a-3p function assay}

Recombinant lentiviral expression vectors were recruited to establish lenti-miR-29a-3p overexpressed vector (miR-29a-3p-enhancer), lenti-miR-29a-3p deregulated vector (miR-29a-3p-inhibitor) and negative control vectors (enhancer-nc and inhibitor-nc) (RiboBio, Guangzhou, China).

Male Wistar rats, fed with HFD for 8 weeks, were used in this experiment. These recombinant lentiviral expression vectors were intramuscularly injected into the femoral metaphysis region ( $n=8$ in each group). 3 days after injection, implants were inserted consistent with the above method. 10 days after implantation, all animals were sacrificed and $1 \mathrm{~mm}$ bone tissues around implants were obtained. HE stained hard tissue slices and qRT-PCR methods were performed according to the previously described protocols.

Table 1 Primers used for qRT-PCR

\begin{tabular}{lll}
\hline Gene & $\begin{array}{l}\text { Forward primer sequence } \\
\left(\mathbf{5}^{\prime} \mathbf{-} \mathbf{3}^{\prime} \mathbf{)}\right.\end{array}$ & $\begin{array}{l}\text { Reverse primer sequence } \\
\left(\mathbf{5}^{\prime} \mathbf{-} \mathbf{3}^{\prime} \mathbf{)}\right.\end{array}$ \\
\hline ALP & TGAGCGACACGGACAAGAAG & GCCTGGTAGTGTTGTGGCAT \\
Runx2 & CACAAGTGCGGTGCAAACTT & AATGACTCGGTTGGTCTCGG \\
Dvl2 & TCCACCATTACCCCCTTGC & GCCATGCTCACTGCTGTCT \\
Fzd4 & GGAAGGACCAGGTGACGAAG & GGAATATGATGGGGCGCTCA \\
GAPDH & TGATGGGTGTGAACCACGAG & CTGATGGGTGTGACCACGAG \\
miR-29a-3p & UAACCGAUUUCAAAUGGUGCUA \\
\hline
\end{tabular}

\section{Biological and functional assays in vitro} BMSCs culture, identification and induction

BMSCs were isolated from male Wistar rat (3 weeks old) and cultured as described previously [40]. BMSCs $(1 \times 106)$ at passage 3 were incubated with antibodies against mouse CD11, CD11b, CD44, CD45, CD90 (BioLegend, USA) and CD29 (eBioscience, USA), fluorescence-Activated Cell Sorting (FACS) was performed by using CXP Analysis 2.1 software (Beckman Coulter, USA). BMSCs of passage 3 were randomly divided into control and experiment groups, control group was induced in osteogenic medium (10\% FBS $\alpha$-MEM culture medium, $501 \mathrm{~g} / \mathrm{ml}$ ascorbic acid, $10 \mathrm{nM}$ dexamethasone, and $10 \mathrm{mM}$ b-glycerophosphatase) and experiment group cultured in HF osteogenic medium (Xingzhi Technology, Guangzhou, China).

\section{Immunofluorescence analysis}

Immunofluorescence analysis was performed to observe the location of ALP and Runx2 proteins and cytoskeletal structures of BMSCs. BMSCs were cultured and fixed in a 6-well culture board and incubated with antibodies specific for ALP (1:1000, Abcam, USA) or Runx2 (1:1000, Abcam, USA). After incubating with goat anti-mouse IgG (1:1000, Abcam, USA), the cystoskeleton was stained by phalloidin (1:200, Solarbio, China) and the nuclei was stained by adding DAPI (1:1000, Invitrogen, D3571). Finally the cells were observed with confocal laser scanning microscope (CLSM, LSM 780, CalZeissAG, Germany), nuclei were counted conducted by ZEISS ZEN SYSTEM 2012 software.

\section{Alkaline phosphatase (ALP) staining and ALP activity assays}

ALP staining and ALP activity assays were used to analyze ALP activity of BMSCs. 7, 14 days after osteogenic induction, BMSCs were washed with PBS and fixed in $4 \%$ paraformaldehyde for $20 \mathrm{~min}$, and stained by BCIP/ NBT ALP Color Development Kit (Beyotime, Shanghai, China) for $15 \mathrm{~min}$ in the dark. After thorough washing with PBS, images were taken using a microscope (Olympus, Japan). An ALP Colorimetric Assay Kit (Jiancheng Biotechnology, Nanjing, China) was used to quantify the ALP expression in control and experiment groups. The data were normalized to the corresponding total protein contents, which were determined using Enhanced BCA Protein Assay Kit (Beyotime Institute of Biotechnology, China).

\section{Alizarin red S (ARS) staining}

BMSCs of control and experiment groups were induced for 28 days, while BMSCs of non-induced group received no induction. ARS staining was conducted to assess the mineralization of BMSCs. The cells were fixed in $2 \%$ 
formaldehyde solution, washed with $\mathrm{ddH}_{2} \mathrm{O}$ and then stained for Ca deposit using 0.5\% ARS (pH 4.0) (Sigma, Germany) for $20 \mathrm{~min}$. Then inverted microscope was used to observe.

\section{Oil red $O$ staining}

To assess the adipogenesis capability of BMSCs, cells (14 days after induction or non-induced) were stained with Oil Red O (Sigma, China) at $37^{\circ} \mathrm{C}$ for $30 \mathrm{~min}$, rinsed with $75 \%$ alcohol until the intercellular substance was clear. Cells were then observed and photographed under an inverted microscope.

\section{$R T-P C R$}

$3,5,7,14$ days after induction, cells of control and experiment groups were collected. qRT-PCR was conducted to analyze ALP, Runx2 mRNA and miR29a-3p expressions, and the methods were consistent with the above.

\section{Western blotting}

Cells of these two groups were gathered on day 3, 5, 7, 14 after induction. Total proteins were extracted and quantified by the BCA Protein Quantitation Kit (Beyotime Biotechnology, China). 0.05ug of protein from each sample were loaded on SDS-PAGE gel and then transferred to PVDF membranes (Millipore, Billerica, MA, USA). Transferred membrane was immunoblotted with primary antibodies (Table 2). After incubation with secondary antibody (goat anti-rabbit IgG, Abcam, USA), protein levels were detected using the Western-Light chemiluminescent detection system (Peiqing, Shanghai, China).

\section{Lenti-vectors transfection, miR-29a-3p function analysis in vitro}

BMSCs were transfected with different concentrations of FAM-siRNA when cells reached $30-50 \%$ confluency. 24,48 and $72 \mathrm{~h}$ after incubation, we analyzed the absorbance at $450 \mathrm{~nm}$ to detect the optimal concentration. MiR29a-3p-enhancer, miR-29a-3p-inhibitor and enhancer/ inhibitor-nc (RiboBio, Guangzhou, China) were transfected into BMSCs respectively. After 48 h, ALP staining was performed to demonstrate ALP activities of these four groups. RT-PCR and Western blotting were used to

Table 2 Antibodies used for western blotting

\begin{tabular}{lll}
\hline Name & Description & Manufacturer \\
\hline Anti-ALP & Rabbit monoclonal, 140 kDa & CST (\#3192) \\
Anti-Runx2 & Rabbit monoclonal, 170 kDa & CST (\#3179) \\
Anti-Dvl2 & Mouse monoclonal, 65 kDa & Abcam (ab181770) \\
Anti-Fzd4 & Rabbit monoclonal, 70 kDa & CSB-PA706537 \\
\hline
\end{tabular}

detect the mRNA and protein levels expressions of miR29a-3p, ALP, Runx2, Fzd4 and Dvl2.

\section{Luciferase reporter assay}

Luciferase reporter assay was performed to detect whether miR-29a-3p targetly regulate Dvl2 and Fzd4. Luciferase reporters were generated based on the pMIRREPORT vector (Sangon Biotech, Shanghai, China). The 3'-UTR sequence of $\mathrm{Dvl} 2 / \mathrm{Fzd} 4$ and the mutant of these sequences, containing the predicted miR-29a-3p binding site, were cloned into the pMIR-REPORT vector, respectively. The luciferase reporter was co-transfected with miR-29a-3p enhancer, miR-29a-3p-mut enhancer, or miR-negative control (miR-NC) into 293T cells by Lipofectamine 3000 according to the manufacturer's guidelines. The relative luciferase activity was measured with the Dual-Luciferase Reporter Assay System Kit (Beyotime, China). The experiments were performed independently in triplicate.

\section{Statistical analysis}

All the data were analyzed by Graphpad Prism 7.0 software (GraphPad Software Inc., La Jolla, CA, USA). The mean differences between the two groups were compared by t-test, and the mean differences among three or more groups were compared by analyzed by one-way ANOVA. $\mathrm{P}<0.05$ from a two-tailed test was considered significant.

\section{Result}

\section{Hyperlipidemia hindered osseointegration and reduced} miR-29a-3p expression

To determine the roles of hyperlipidemia in osseointegration and miR-29a-3p expression in vivo, hyperlipidemia animal models were established by HFD. Serum lipid levels of the animals from the two groups are listed in Table 3. TC increased by about twofolds, and TG and LDL obtained significantly increase in the HF group comparing to the normal group $(P<0.05)$, this result confirmed that our model successfully resembled hyperlipidemia in HF groups. HE images showed the bone mass, bone density and new bone volume were significantly reduced, and bone tissue was sparse on HF group, while implants were tightly integrated with the surrounding

Table 3 The serum TC, TG, HDL and LDL of two groups

\begin{tabular}{lllll}
\hline & TC & TG & HDL & LDL \\
\hline Normal range & $1.64 \pm 0.21$ & $1.01 \pm 0.46$ & $0.91 \pm 0.15$ & $0.24 \pm 0.05$ \\
Control group & $1.45 \pm 0.22$ & $0.73 \pm 0.12$ & $1.10 \pm 0.16$ & $0.29 \pm 0.66$ \\
Experiment group & $3.45 \pm 0.65^{*}$ & $1.74 \pm 0.68^{*}$ & $1.07 \pm 0.22^{*}$ & $0.65 \pm 0.12^{*}$ \\
\hline
\end{tabular}

Values are mean $\pm S D$ in $\mathrm{mmol} / \mathrm{l}, * P<0.05$ was considered statistically significant 
bone tissues on normal group. Moreover, many lightly stained osteoblasts were visible under high magnification in the normal group (Fig. 1a).

qRT-PCR results demonstrated that ALP mRNA and miR-29a-3p up-regulated on day 5 , peaked on day 10 , and returned to normal on day 20, while Runx 2 mRNA increased on day 5 , peaked on day 15 , and returned to normal on day 20. As we expected, the expressions of these genes were significantly decreased in HF group compared to the normal group, especially on day 10 (Fig. 1b). Taken together, we demonstrated that hyperlipidemia hindered osseointegration was correlated with deregulated miR-29a-3p expression.

\section{MiR-29a-3p overexpression enforced osseointegration and miR-29a-3p inhibitor repressed osseointegration} in vivo

To further explore the function of miR-29a-3p on osseointegration, miR-29a-3p gain- and reduce-of-function assays were performed in hyperlipidemia models. Implants were surrounded by abundant newly formed woven bone, and bone mass, new bone volume was increased on miR-29a-3p overexpressed group, while a bare amount of bone tissue was observed in the implantbone interface of miR-29a-3p inhibited group (Fig. 2a, b). qRT-PCR results demonstrated that miR-29a-3p, ALP and Runx 2 mRNAs were dramatically up-regulated in miR-29a-3p-enhancer treated group (miR29a3p 34-folds, ALP 5.7-folds, Runx2 7.6-folds) compared to the control group. Conversely, these genes were significantly declined when transfected with miR-29a-3pinhibitor (miR-29a-3p 0.36-folds, ALP 0.43-folds, Runx2 0.62 -folds) compared to the inhibitor-nc group (Fig. 2c). Collectively, these results suggested that hyperlipidemia impaired osseointegration and reduced miR-29a-3p expression, while with the expression of miR-29a-3p upregulated, osseointegration was promoted.

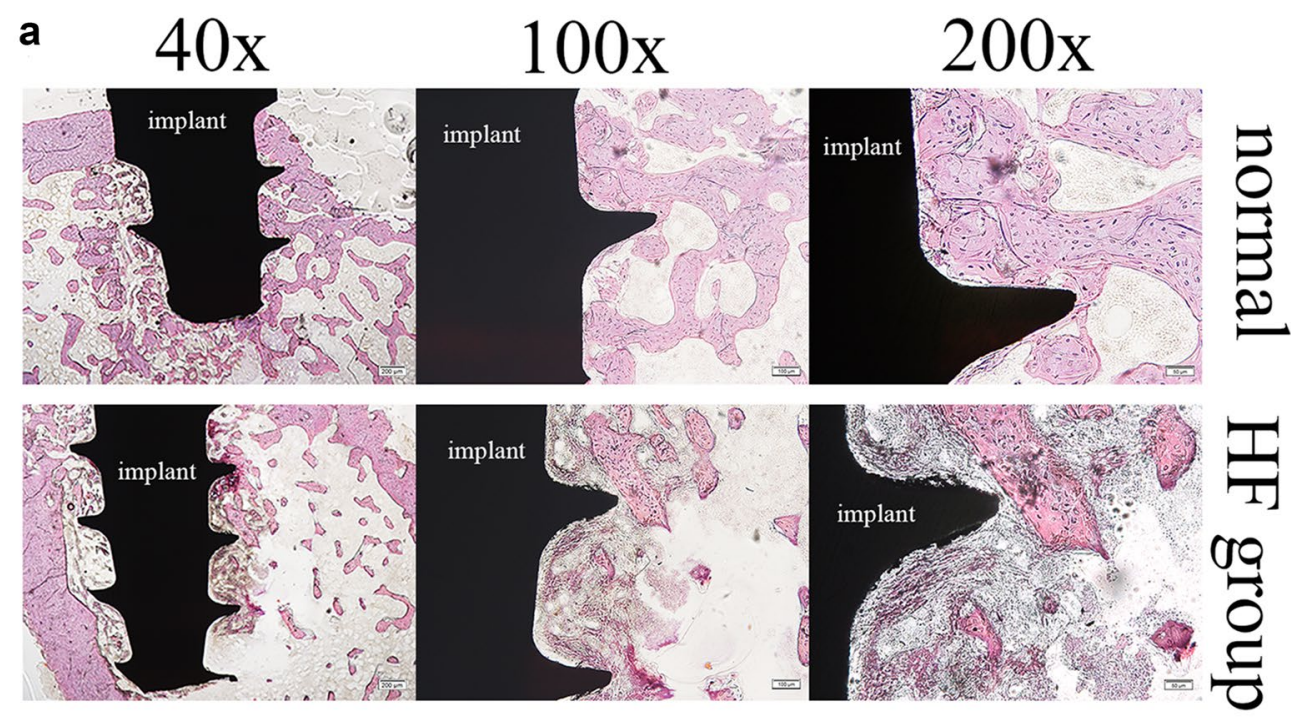

b

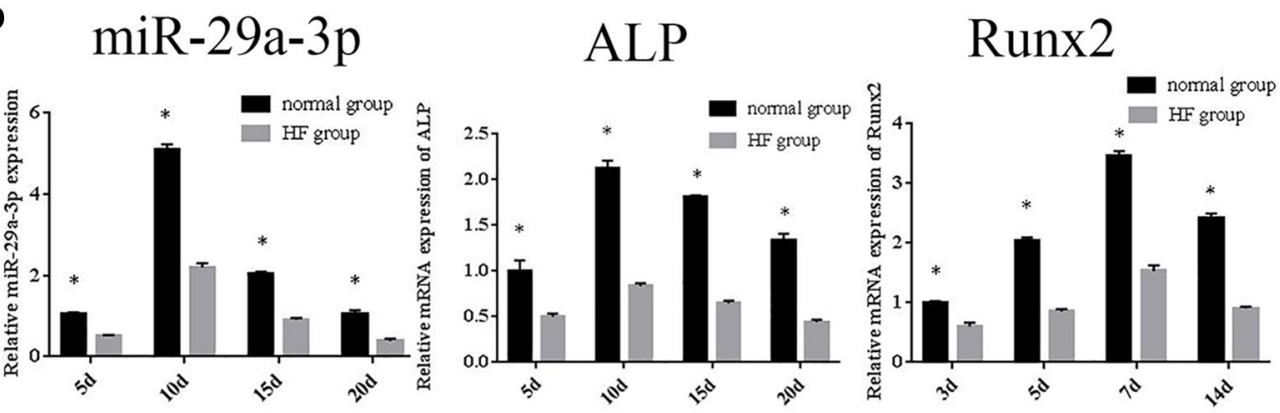

Fig. 1 MiR-29a-3p expression was deregulated in hyperlipidemia rats along with impaired osseointegration. a HE stained hard tissue slices were used to assess the osseointegration of implant periphery bone both of normal and HF groups. $\mathbf{b}$ qRT-PCR analysis was used to determine the relative expression levels of miR-29a-3p, ALP mRNA and Runx2 mRNA in peri-implant bone tissues of HF group and normal group. Results were represented as mean $\pm S D$. ${ }^{*} P<0.05$ 


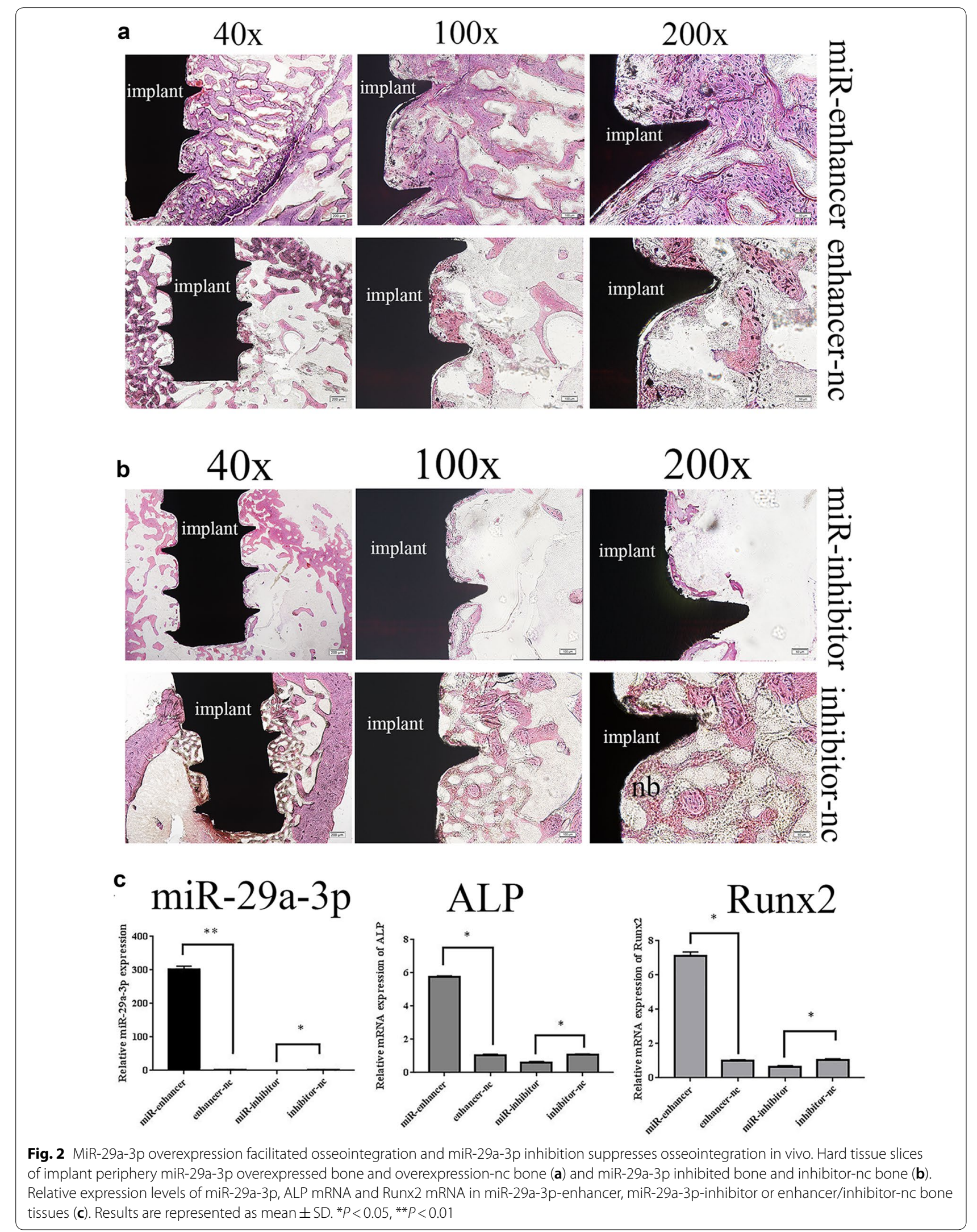


Hyperlipidemia weakened BMSCs' osteogenesis in vitro Given the adverse effect of hyperlipidemia on osseointegration in rats, next we investigated the functional role of hyperlipidemia in vitro. To assess the purity of rat BMSCs, we used FACS to detect the markers of BMSCs. Our results showed that rat BMSCs expressed CD44, CD90 and CD29 (+, positive rate $>95 \%$ ), CD11, CD11b and CD45 ( - , positive rate $<5 \%$ ), indicating that BMSCs were of high purity (Fig. 3a).

Cytoskeletons, related to bone matrix deposit, are vital for osteogenesis [41]. Immunofluorescence staining was exerted to show the cytoskeletal structure and the locations of ALP and Runx 2 proteins in cells. The cytoskeletal structure of osteoblast in control group tends to be dense and fine ordered, while the experiment group gained sparse and disordered cytoskeletal structure. Moreover, ALP and Runx2 proteins were widely distributed in the cytoplasm and their expressions were significantly weakened in experiment group compared to the control group (Fig. 3b, c).

BMSCs' osteogenic differentiation is correlated with the increased expression of intracellular ALP. To investigate the biological and functional roles of hyperlipidemia in osteogenic differentiation in vitro, ALP staining and ALP activity assays were conducted in BMSCs 7 days after induction. ALP staining showed that the activity of ALP on day 7 was significantly decreased in experiment group in comparison to control group (Fig. 3d). Consistently, ALP activity assays showed that ALP activity in experiment group was considerably lower than the control group both on day 7 and 14 (Fig. 3d).

Osteogenesis was accompanied by mineralization. ARS staining was performed to assess the mineralization function of osteoblasts. The density, volume and quantity of mineralized nodules were reduced in the experiment group in comparison to the control group (Fig. 3e), while little mineralized nodules were observed in the noninduced group. These results indicated that there was a lower level of mineralization of osteoblasts in the experiment group.

Additionally, high standard adipogenesis is correlated with low standard osteogenesis [1-3]. Oil red O staining was used to examine adipogenesis of non-induced, control and experiment groups. A large amount of lipid drops were observed in the experiment group, while only a few lipid drops existed in the control group and barely no lipid drops were observed in the non-induced group (Fig. 3f). These results demonstrated that hyperlipidemia facilitates adipogenesis, which further confirmed that hyperlipidemia impaired osteogenic differentiation of BMSCs.
Hyperlipidemia reduced the expression of miR-29a-3p during osteogenesis in intro

qRT-PCR showed miR-29a-3p and ALP, Runx2 mRNAs increased on day 3 , summited on day 7 , and then decreased to normal on day 14. Importantly, the gene expressions were lower in the experiment group than in the control group, especially on day 7 (miR-29a-3p 0.2fold, ALP 0.48-folds, Runx2 0.35-folds) (Fig. 3g). Similarly, Western blotting showed the reduction of ALP and Runx2 proteins in the experiment group in comparison to the control group (Fig. 3h). These results indicated that miR-29a-3p was suppressed by hyperlipidemia during BMSC osteogenesis.

\section{Overexpressed miR-29a-3p increased osteogenesis} while downexpressed miR-29a-3p decreased osteogenesis ALP staining delineated that ALP activity was greatly increased in cells transfected with miR-29a-3p-enhancer and suppressed by miR-29a-3p inhibitor (Fig. 4a). qRTPCR showed the up-regulation of miR-29a-3p, ALP and Runx2 mRNA (miR-29a-3p 8.1-folds, ALP 5.8-folds, Runx2 3.0-folds) of BMSCs transfected with miR-29a$3 p$-enhancer in comparison to the enhancer-nc group, while miR-29a-3p-inhibitor down-regulated these genes (miR-29a-3p 0.31-fold, ALP 0.057-fold, Runx2 0.22-folds) compared to the inhibitor-nc group. As we expect, western blotting also showed that ALP, Runx2 proteins were up-regulated in the miR-enhancer group and down-regulated in the miR-inhibitor group (Fig. 4b, c). Together, overexpression of miR-29a-3p promoted osteogenesis and deregulated miR-29a-3p suppressed osteogenesis in vitro.

\section{MiR-29a-3p directly suppressed Dvl2 and Fzd4 in osteogenesis}

By using different databases (miRDB, Target Scan, MicroRNA.org, Microcosm Targets), we predicted that miR-29a-3p could directly suppress Dvl2 and Fzd4. Firstly, qRT-PCR demonstrated that Dvl2 mRNA started to ascend on day 5, submitted on day 15 , and returned to normal on day 20 both in HF and normal rats, and Dvl2 mRNA was slightly suppressed in the HF group compared to the normal group on each time point (Fig. 5a). Fzd4 mRNA in the normal group was dramatically declined from day 5 to day 10, then upregulated until day 15 , and returned to normal on day 20. Fzd4 mRNA on HF group started to increase on day 5 , peaked on day 15 , and returned to normal on day 20 (Fig. 5a). Next, to verify whether miR-29a-3p could directly bind to Dvl2 and Fzd4, qRT-PCR, western blotting were conducted in BMSCs and dual luciferase reporter gene assay was 

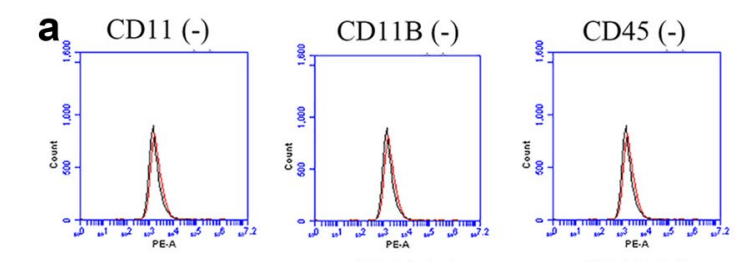

d
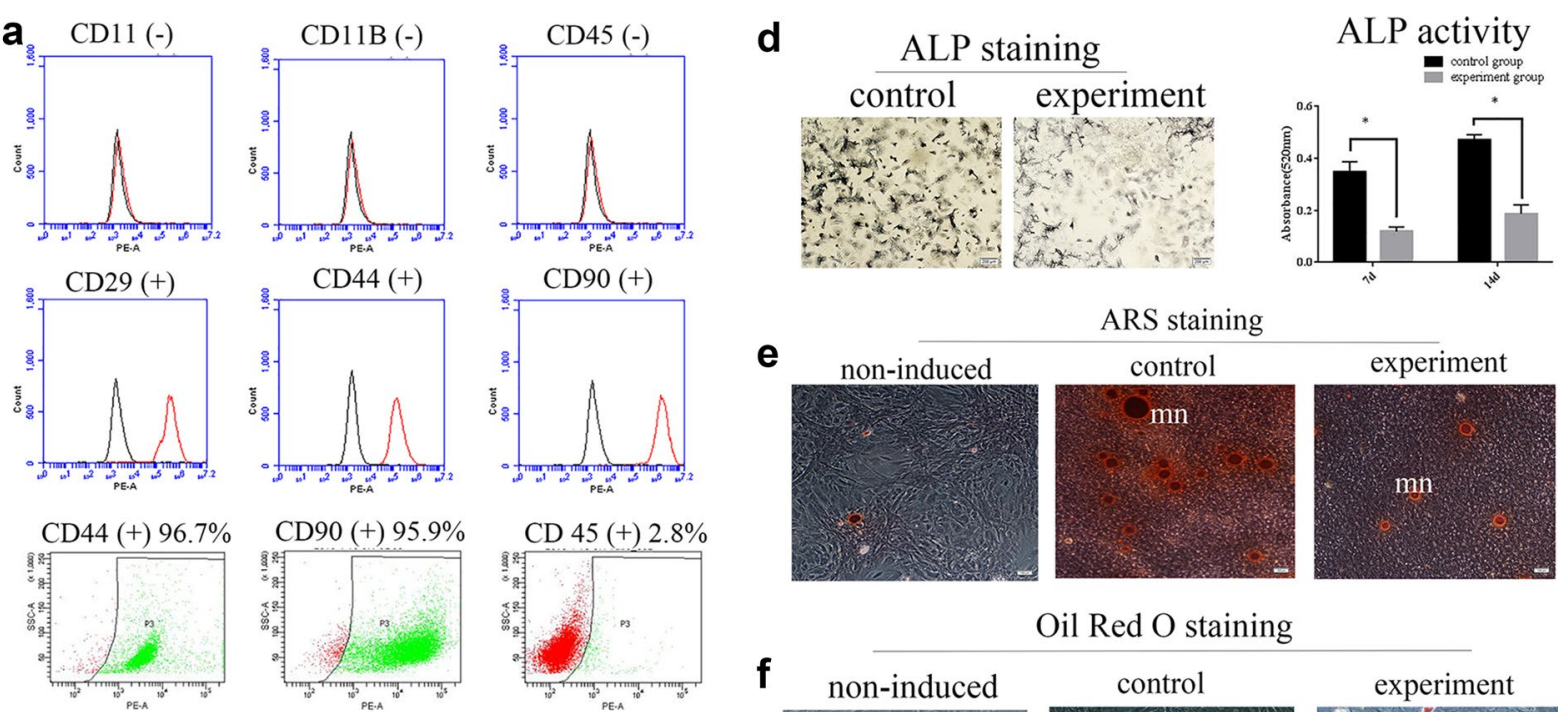

ARS staining

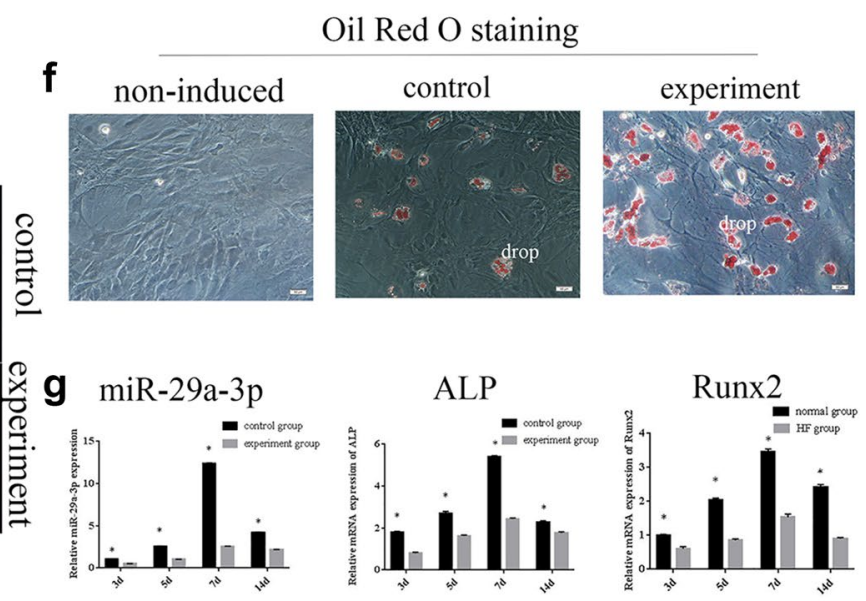

c DAPI antil-Fzd4 Phalloidin Merge
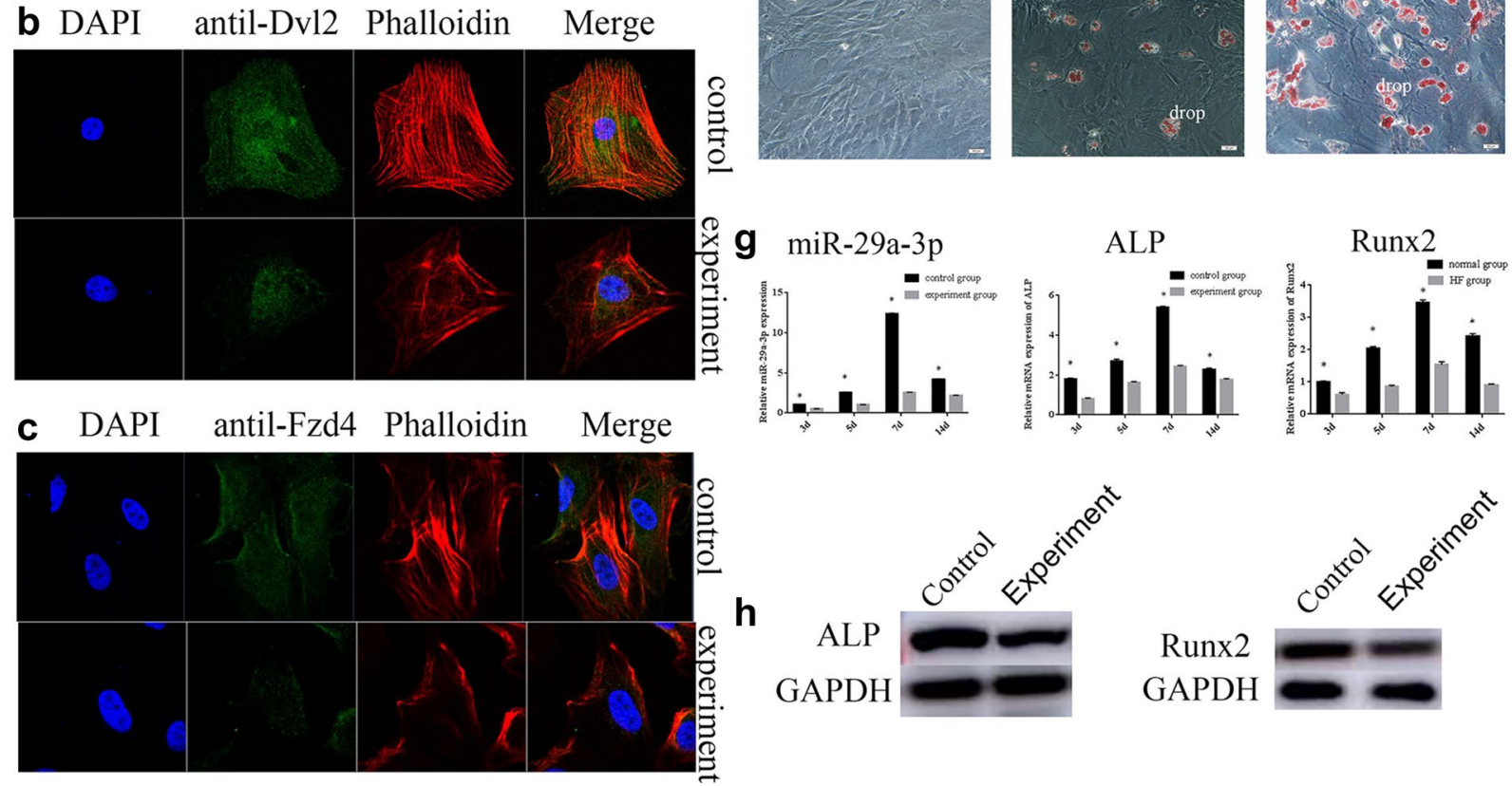

Fig. 3 MiR-29a-3p expression was decreased in experiment group correlated with compromised osteogenic differentiation. a FACS was performed to identify BMSCs and assess the purity of BMSCs. Immunofluorescence was carried out to show the cytoskeletal structures and the introcellular location of ALP proteins (b), and Runx2 proteins (c). $\mathbf{d}$ ALP staining and ALP activity assays were applied to detect osteogenic differentiation. $\mathbf{e}$ ARS staining was used to determine the mineralization of osteoblasts, $\mathrm{mn}$ : mineralized nodule. $\mathbf{f}$ Oil red $\mathrm{O}$ staining was conducted to analyze the adipogenesis of osteoblasts, drop: lipid drop. g Relative expression levels of miR-29a-3p, ALP mRNA and Runx2 mRNA in control and experiment groups. $\mathbf{h}$ Western blotting was used to detect ALP and Runx2 proteins in control and experiment groups. Results are represented as mean \pm SD of three independent experiments. ${ }^{*} P<0.05$

conducted in 293T cells. qRT-PCR and Western blotting demonstrated that overexpressed miR-29a-3p repressed the Dvl2 and Fzd4 mRNA and protein expressions, while miR-29a-3p-inhibitor enhanced their expressions (Fig. 5b, c). Furthermore, dual luciferase reporter assays revealed that miR-29a-3p-enhancer significantly decreased the luciferase activity of wild-type $3^{\prime}$-UTR compared to the miR-NC group. Strikingly, this inhibition was revoked when the miRNA response element was mutated (Fig. 5d). All these data suggested that miR29a-3p directly interacted with Dvl2 and Fzd4 3'-UTR promoter region to regulate its expression. 

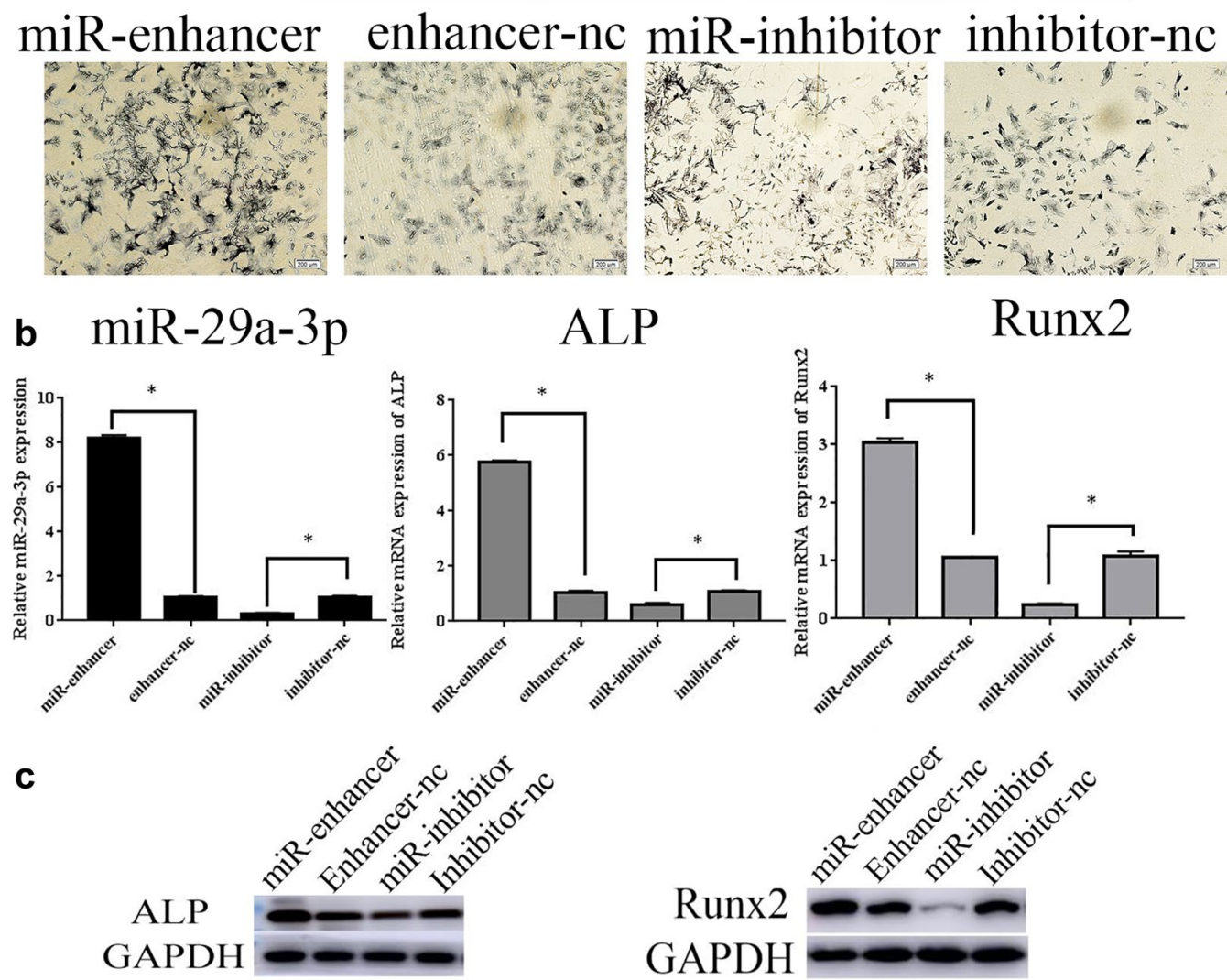

Fig. 4 Overexpressed miR-29a-3p forced osteogenic differentiation and miR-29a-3p inhibitor reduced differentiation in vitro. BMSCs were transfected with miR-29a-3p enhancer, enhancer-nc, miR-29a-3p inhibitor, or inhibitor-nc, followed by the measurement of osteogenic differentiation by ALP staining (a), miR-29a-3p, ALP mRNA and Runx2 mRNA (b), and ALP and Runx2 proteins (c). Results are represented as mean $\pm S D$. ${ }^{*} P<0.05$

\section{Discussion}

Previous studies have discovered that hyperlipidemia was closely associated with bone and bone disease and a variety of miRNAs have been demonstrated to affect osteogenesis through modulating osteogenic differentiation, bone matrix deposition, cytoskeleton formation and osteoclast metabolism $[1-3,20-24]$. MiR-29a-3p was previously suggested to facilitate osteogenic differentiation through activating Wnt/ $\beta$-catenin signal pathway [27]. However, further explorations on the biological significance of hyperlipidemia in osseointegration, as well as the underlying mechanisms of miR-29a-3p in regulating osseointegration and osseointegration are required.

Keuroghlian et al. [3] demonstrated that hyperlipidemia significantly increased implant loss and decreased the formation and strength of the bone-to-implant interface in the mouse femur, which were supported by such observations that increased implant failure, decreased osseointegration, and poor mechanical strength were associated with untreated hyperlipidemia in patients. In addition, Pirih et al. delineated that hyperlipidemia induces secondary hyperparathyroidism and impairs bone regeneration and mechanical strength [2]. Here, we found that osseointegration was significantly hindered by hyperlipidemia in a rat model, further confirmed previous reports in human and mice.

A great many of studies highlighted that miR-29a-3p modulate BMSCs proliferation and differentiation. MiR29a-3p facilitated transplantation of BMSCs to alleviate pelvic floor dysfunction by repressing elastin [4]. In addition, miR-29a-3p was significantly deregulated in the serum of patients with low bone mass compared with controls [42]. Moreover, miR-29a-3p expression increased during osteoblasts mature process correlated with activated Wnt signaling [27]. In the current study, we found that decreased miR-29a-3p in hyperlipidemia rats correlated with impaired osseointegration. Moreover, subsequent gain- and reduce-of-function experiments 

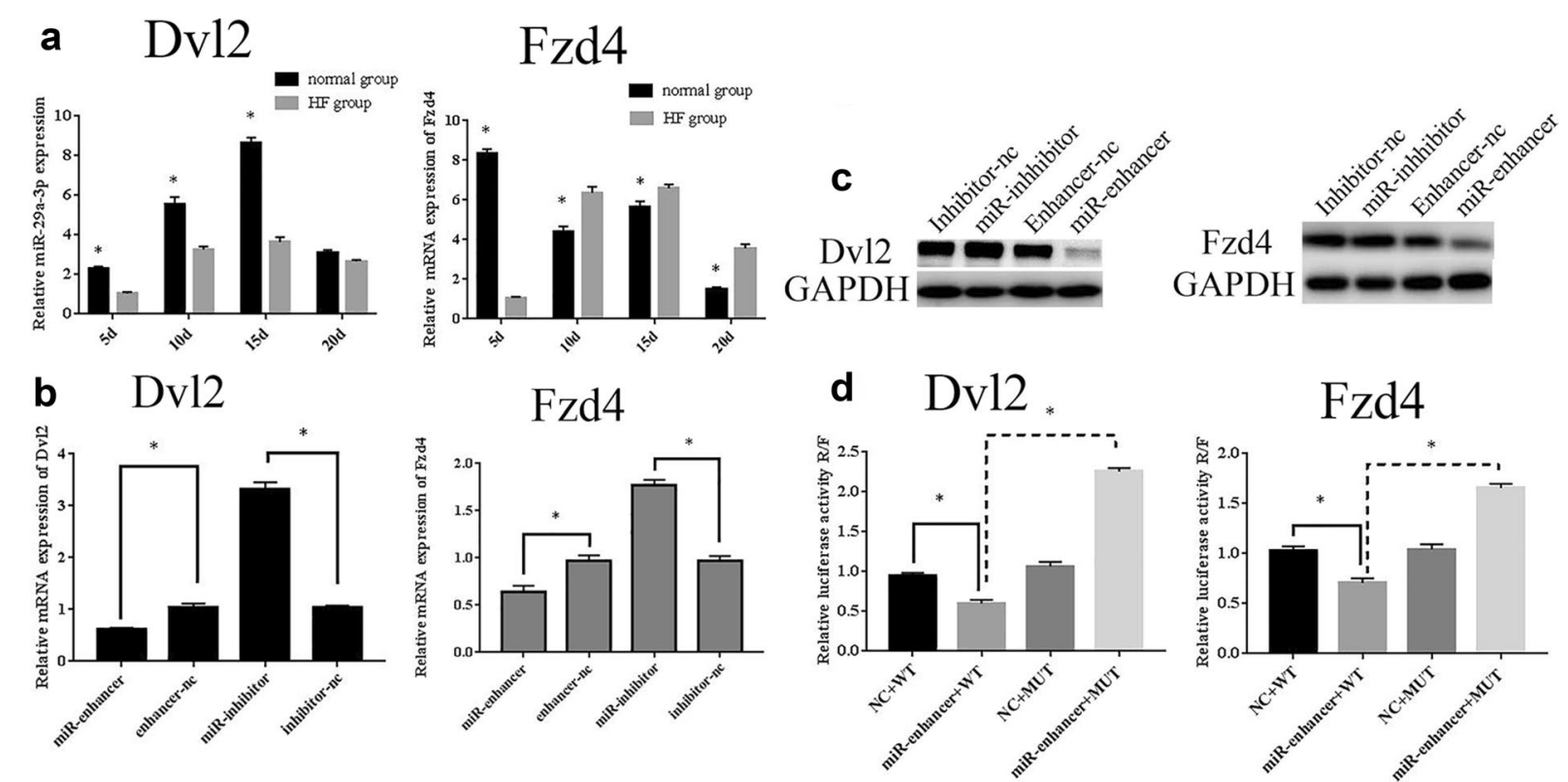

Fig. 5 miR-29a-3p directly suppressed Dvl2 and Fzd4 expression by binding to their $3^{\prime}-U T R$. a mRNA levels of Dvl2 and Fzd4 in peri-implant bone tissue in hyperlipidemia and normal rat models. The mRNA levels of Dvl2 and Fzd4 (b) and protein levels of Dvl2 and Fzd4 (c) in BMSCs transfected with miR-29a-3p enhancer, enhancer-nc, miR-29a-3p inhibitor, or inhibitor-nc. $\mathbf{d}$ Luciferase reporters assay was performed in 293T cells after transfected with miR-29a-3p-WT enhancer reporter, miR29a-3p-MUT enhancer reporter or miR-WT/MUT NC. Results are represented as mean \pm SD. ${ }^{*} P<0.05$

demonstrated that miR-29a-3p overexpression enhanced osseointegration and osteoblastic differentiation, while inhibition of miR-29a-3p obtained opposite result. Our previous study demonstrated that miR-29c-3p promoted osteogenic differentiation of BMSCs by directly suppressing Dvl2 in the $\mathrm{Wnt} / \beta$-catenin signal pathway-related [40], which played critical roles in bone formation, reconstruction and maintenance [43-46].

Wnt/ $\beta$-catenin pathway enhanced bone formation by up-regulating osteogenic differentiation via promoting the expression of osteogenesis related genes, such as Runx2, ALP and OPN (osteoprotegerin) [12-15, 47]. In addition, Wnt/ $\beta$-catenin pathway enhanced the activity through binding to the 7-transmembrane frizzled (Fzd) receptor and low-density lipoprotein 5 and 6 (LRP5/6) co-receptors, which in turn recruited the most proximal signaling intermediate, Dvl, to the plasma membrane [43, 48]. Based on this, we further tested whether miR-29a-3p could regulate osseointegration through suppressing predicted targets Dvl2 and Fzd4. Indeed, we confirmed that miR-29a-3p could suppress Dvl2 and Fzd4 through directly binding their $3^{\prime}$-UTRs. To our knowledge, this was the first experiment to verify the direct inhibition of miR-29a-3p to Dvl2 and Fzd4 during osseointegration.

Dvl2 played a critical role in the Wnt signal transduction pathways $[40,49,50]$. The activity of Dvl2 was up-regulated upon its phosphorylation [51], and down-regulated by ubiquitination [52]. We found in our previous study that hyperlipidemia reduced osseointegration in rats via suppressing the expression of phosphor-Dvl2 as well as promoting expression of ubiquitinated-Dvl2. In this study, we showed that there was a significant reduction of Dvl2 during osseointegration in normal rats in comparison to hyperlipidemia rats. What is more, the expression of Fzd4 fluctuated in the normal and HF groups in vivo, and Fzd4 expression was significantly higher at the middle stage of osseointegration in hyperlipidemia rats than in normal rats. Several studies elucidated that Fzd4 plays a role in osteogenesis. For example, Long et al. demonstrated that miR139-5p inhibited BMSCs' osteogenesis via directly targeting Fzd4 and $\beta$-catenin [53]. In addition, Fzd4 was engaged in Wnt5a mediated orthodontic force induced bone remodeling via activating Wnt/ $\beta$-catenin signaling pathway [54]. Furthermore, Dvl2 could recruit $\beta$-Arrestin 2, ubiquitous cellular scaffolding proteins, to mediate Wnt5a-stimulated endocytosis of Fzd4 [48], further indicating the significant importance of the interaction between Dvl2 and Fzd4 during the activation of Wnt signal pathway.

Nevertheless, though the present study has demonstrated that Dvl2 and Fzd4 were the direct target of miR-29a-3p, the underlying mechanisms of miR-29a-3p targeting Dvl2 and Fzd4, and the interactions between Dvl2 and Fzd4 remained unclear. Further studies are needed to elucidate these molecular mechanisms. 


\section{Conclusion}

In conclusion, our results showed the adverse effect of hyperlipidemia on osseointegration in rats. Moreover, we demonstrated that miR-29a-3p enforced osseointegration by targeting Dvl2 and Fzd 4 in the Wnt/ $\beta$-catenin pathway, and this enforcement could be impaired by hyperlipidemia.

\section{Abbreviations}

BMSCs: bone marrow mesenchymal stem cells; miRNAs: microRNAs; HF: highfat; HFD: high-fat diet; miR-29a-3p: microRNA-29a-3p; ALP: alkaline phosphatase; Runx2: runt-related transcription factor 2; Dvl2: dishevelled 2; Fzd4: frizzled 4; qRT-PCR: real-time qantitative PCR; PVDF: polyvinylidene difluoride; FACS: fluorescence-activated cell sorting.

\section{Authors' contributions}

$\mathrm{FL}$ conceived and designed the experiments in the manuscript. FL, FFL, and JZX performed the experiments. FL and ZFW analyzed data and plotted the graphs for figures. FL wrote the manuscript. All authors read and approved the final manuscript.

\begin{abstract}
Author details
${ }^{1}$ Department of Prosthodontics, School of Stomatology, Shandong University, Jinan 250000, China. ${ }^{2}$ Shandong Provincial Key Laboratory of Oral Tissue Regeneration, 44-1 West Wenhua Street, Jinan 250012, Shandong, China. ${ }^{3}$ Department of Pediatric Dentistry, School of Stomatology, Shandong University, Jinan 250000, China. ${ }^{4}$ Department of Orthodontics, Herman Ostrow School of Dentistry, Los Angeles, CA 90089, USA. ${ }^{5}$ Center for Craniofacial Molecular Biology, Herman Ostrow School of Dentistry, Los Angeles 90033, USA. ${ }^{6}$ Department of Implantology, Stomatological Hospital of Nanyang, Nanyang 473000, China. ${ }^{7}$ Department of Prosthodontics, School of Dentistry, Shandong University, Jinan, China.
\end{abstract}

\section{Acknowledgements}

We would like to thank all the colleagues in our research team.

\section{Competing interests}

The authors declare that they have no competing interests.

\section{Availability of data and materials}

All relevant data are within this published paper.

\section{Consent for publication}

Not applicable.

\section{Ethics approval and consent to participate}

All experimental procedures involving animals were done in accordance with the institutional ethical guidelines for animal experiments and were approved by Shandong University Ethics Committee (Grant No. GD201615).

\section{Funding}

This study was funded by National Natural Science Foundation of China 81671025 (Beijing, China), and Foundation of Department of Science and Technology of Shandong Province 2015GSF118186 (Ji'nan, China), Natural Science Foundation of Shandong Province ZR2018ZB0105 (Ji'nan, China).

\section{Publisher's Note}

Springer Nature remains neutral with regard to jurisdictional claims in published maps and institutional affiliations.

Received: 30 May 2018 Accepted: 22 October 2018

Published online: 26 October 2018

\section{References}

1. Magán-Fernández A, Papay-Ramírez L, Tomás J, Marfil-Álvarez R, Rizzo M, Bravo M, Mesa F. Association of simvastatin and hyperlipidemia with periodontal status and bone metabolism markers. J Periodontol. 2014;85:1408-15.

2. Pirih F, Lu J, Ye F, Bezouglaia O, Atti E, Ascenzi MG, Tetradis S, Demer L, Aghaloo T, Tintut Y. Adverse effects of hyperlipidemia on bone regeneration and strength. J Bone Miner Res. 2012;27:309-18.

3. Keuroghlian A, Barroso AD, Kirikian G, Bezouglaia O, Tintut Y, Tetradis S, Moy P, Pirih F, Aghaloo T. The effects of hyperlipidemia on implant osseointegration in the mouse femur. J Oral Implantol. 2015;41:7-11.

4. Jin M, Wu Y, Wang J, Ye W, Wang L, Yin P, Liu W, Pan C, Hua X. MicroRNA-29 facilitates transplantation of bone marrow-derived mesenchymal stem cellsto alleviate pelvic floor dysfunction by repressing elastin. Stem Cell Res Ther. 2016;7:167.

5. Smeets R, Stadlinger B, Schwarz F, Beck-Broichsitter B, Jung O, Precht C, Kloss F, Gröbe A, Heiland M, Ebker T. Impact of dental implant surface modifications on osseointegration. Biomed Res Int. 2016. https://doi. org/10.1155/2016/6285620.

6. Ahmed HM, Miller M, Nasir K, McEvoy JW, Herrington D, Blumenthal RS, Blaha MJ. Primary low level of high-density lipoprotein cholesterol and risks of coronary heart disease, cardiovascular disease, and death: results from the multi-ethnic study of atherosclerosis. Am J Epidemiol. 2016;183:875-83.

7. Matziolis D, Tuischer J, Matziolis G, Kasper G, Duda G, Perka C. Osteogenic predifferentiation of human bone marrow-derived stem cells by short-term mechanical stimulation. Open Orthop J. 2011;5:1-6.

8. Undale AH, Westendorf JJ, Yaszemski MJ, Khosla S. Mesenchymal stem cells for bone repair and metabolic bone diseases. Mayo Clin Proc. 2009;84:893-902

9. Xu QC, Hao PJ, Yu XB, Chen SL, Yu MJ, Zhang J, Yang PS. Hyperlipidemia compromises homing efficiency of systemically transplanted BMSCs and inhibits bone regeneration. Int J Clin Exp Pathol. 2014;7:1580-7.

10. Yu ZD, Gauthier P, Tran QT, El-Ayachi I, Bhatti FU, Bahabri R, Al-Habib $M$, Huang GT. Differential properties of human ALP+ periodontal ligament stem cells vs their ALP - counterparts. J Stem Cell Res Ther. 2015;5:292.

11. Komaki M, Iwasaki K, Arzate H, Narayanan AS, Izumi Y, et al. Cementum protein 1 (CEMP1) induces a cementoblastic phenotype and reduces osteogenic differentiation in periodontal ligament cells. J Cell Physiol. 2012;227:649-57

12. Tsai MT, Li WJ, Tuan RS, Chang WH. Modulation of osteogenesis in human mesenchymal stem cells by specific pulsed electromagnetic field stimulation. J Orthop Res. 2009;27:1169-74.

13. Liu J, Jin T, Ritchie HH, Smith AJ, Clarkson BH. In vitro differentiation and mineralization of human dental pulp cells induced by dentin extract. Vitro Cell Dev Biol Anim. 2005;41:232-8.

14. Trotter TN, Li M, Pan Q, Peker D, Rowan PD, Li J, Zhan F, Suva L, Javed A, Yang Y. Myeloma cell-derived Runx2 promotes myeloma progression in bone. Blood. 2015;125:3598-608.

15. Bruderer M, Richards RG, Alini M, Stoddart MJ. Role and regulation of Runx2 in osteogenesis. Eur Cell Mater. 2014;28:269-86.

16. Pan H, Li X, Wang J, Zhang K, Yang H, Li Z, Zheng Z, Liu H. LIM mineralization protein-1 enhances bone morphogenetic protein-2-mediated osteogenesis through activation of ERK1/2 MAPK pathway and upregualtion of Runx2 transactivity. J Bone Miner Res. 2015;30:1523-35.

17. Nagel AK, Ball LE. O-GlCNAC modification of the runt-related transcription factor 2 (Runx2) links osteogenesis and nutrient metabolism in bone marrow mesenchymal stem cells. Mol Cell Proteomics. 2014;13:3381-95.

18. Mohr AM, Mott JL. Overview of microRNA biology. Semin Liver Dis. 2015;35:3-11.

19. van Solingen C, Bijkerk R, de Boer HC, Rabelink TJ, van Zonneveld AJ. The role of microRNA-126 in vascular homeostasis. Curr Vasc Pharmacol. 2015;13:341-51.

20. Li Z, Hassan MQ, Jafferji M, Aqeilan Rl, Garzon R, Croce CM, van Wijnen AJ, Stein JL, Stein GS, Lian JB. Biological functions of miR-29b contribute 
to positive regulation of osteoblast differentiation. J Biol Chem. 2009;284:15676-84.

21. Zhou Q, Zhao ZN, Cheng JT, Zhang B, Xu J, Huang F, Zhao RN, Chen YJ. Ibandronate promotes osteogenic differentiation of periodontal ligament stem cells by regulating the expression of microRNAs. Biochem Biophys Res Commun. 2011:404:127-32.

22. Liu Y, Liu W, Hu C, Xue Z, Wang G, Ding B, Luo H, Tang L, Kong X, Chen X, Liu N, Ding Y, Jin Y. MiR-17 modulates osteogenic differentiation through a coherent feed-forward loop in mesenchymal stem cells isolated from periodontal ligaments of patients with periodontitis. Stem Cells. 2011;29:1804-16.

23. Hassan MQ, Gordon JA, Beloti MM, Croce CM, van Wijnen AJ, Stein JL, Stein GS, Lian JB. A network connecting Run×2, SATB2, and the miR23a 27a 24-2 cluster regulates the osteoblast differentiation program. Proc Natl Acad Sci USA. 2010;107:19879-84.

24. Wang $L L$, Huang $Y$, Wang G, Chen SD. The potential role of microRNA-146 in Alzheimer's disease: biomarker or therapeutic target? Med Hypotheses. 2012;78:398-401.

25. Materozzi M, Merlotti D, Gennari L, Bianciardi S. The potential role of miRNAs as new biomarkers for osteoporosis. Int J Endocrinol. 2018;6:e2342860

26. Le LT, Swingler TE, Crowe N, Vincent TL, Barter MJ, Donell ST, Delany AM, Dalmay T, Young DA, Clark IM. The microRNA-29 family in cartilage homeostasis and osteoarthritis. J Mol Med (Berl). 2016;94:583-96.

27. Kapinas K, Kessler CB, Delany AM. miR-29 suppression of osteonectin in osteoblasts: regulation during differentiation and by canonical Wnt signaling. J Cell Biochem. 2009;108:216-24.

28. Monroe DG, McGee-Lawrence ME, Oursler MJ, Westendorf JJ. Update on Wnt signaling in bone cell biology and bone disease. Gene. 2012;15(492):1-18.

29. Wang H, Sun W, Ma J, Pan Y, Wang L, Zhang WB. Biglycan mediates suture expansion osteogenesis via potentiation of $\mathrm{Wnt} / \beta$-catenin signaling. J Biomech. 2015;48:432-40.

30. Zhang $\mathrm{H}, \mathrm{LiH}$. Tricin enhances osteoblastogenesis through the regulation of Wnt/ $\beta$-catenin signaling in human mesenchymal stem cells. Mech Dev. 2018;152:38-43.

31. Gao X, Ge J, Li W, Zhou W, Xu L. LncRNA KCNQ1OT1 promotes osteogenic differentiation to relieve osteolysis via Wnt/ $\beta$-catenin activation. Cell Biosci. 2018;8:19.

32. Yang C, Yifan L, Dan L, Qian Y, Ming-yan J. Bamboo leaf flavones and tea polyphenols show a lipid-lowering effect in a rat model of hyperlipidemia. Drug Res (Stuttg). 2015;65:668-71.

33. Dong $X$, Wang $Z$, Wang $H$, Lan J. The research of dishevelled-2 in dental implant osseointegration of hyperlipidemic rats. Int J Oral Maxillofac Implants. 2018;33:351-6.

34. LiY, He S, Hua Y, Hu J. Effect of osteoporosis on fixation of osseointegrated implants in rats. J Biomed Mater Res B Appl Biomater. 2017;105:2426-32.

35. Eto S, Miyamoto H, Shobuike T, Noda I, Akiyama T, Tsukamoto M, Ueno M, Someya S, Kawano S, Sonohata M, Mawatari M. Silver oxide-containing hydroxyapatite coating supports osteoblast function and enhances implant anchorage strength in rat femur. J Orthop Res. 2015;33:1391-7.

36. Melissa V, Gammons MV, Rutherford TJ, Steinhart Z, Angers S, Bienz M. Essential role of the dishevelled DEP domain in a Wnt-dependent human-cell-based complementation assay. J Cell Sci. 2016;129:3892-902.

37. Mahuzier A, Gaudé HM, Grampa V, Anselme I, Silbermann F, Leroux-Berger M, Delacour D, Ezan J, Montcouquiol M, Saunier S, Schneider-Maunoury
S, Vesque C. Dishevelled stabilization by the ciliopathy protein Rpgrip1 I is essential for planar cell polarity. J Cell Biol. 2012;198:927-40.

38. Wang YS, Chang H, Rattner A, Nathans J. Frizzled receptors in development and disease. Curr Top Dev Biol. 2016;117:113-39.

39. Chen YJ, Zhang Y, Tang JJ, Liu F, Hu Q, Luo CX, Tang JP, Feng H, Zhang JH. Norrin protected blood brain barrier via frizzled $4 / \beta$-catenin pathway after subarachnoid hemorrhage in rats. Stroke. 2015;46:529-36.

40. Huang X, Li DD, Wang ZF, Huang ZF, Dong XF, Li CH, Lan J. Study of microRNAs targeted Dvl2 on the osteoblasts differentiation of rat BMSCs in hyperlipidemia environment. J Cell Phsiol. 2018;233:6758-66.

41. Klein-Nulend J, Bacabac RG, Bakker AD. Mechanical loading and how it affects bone cells: the role of the osteocyte cytoskeleton in maintaining our skeleton. Eur Cell Mater. 2012;24:278-91.

42. Yavropoulou MP, Anastasilakis AD, Makras P, Tsalikakis DG, Grammatiki M, Yovos JG. Expression of microRNAs that regulate bone turnover in the serum of postmenopausal women with low bone mass and vertebral fractures. Eur J Endocrinol. 2017;176:169-76.

43. Mei G, Zou ZL, Fu S, Xia LH, Zhou J, Zhang YT, Tuo YH, Wang Z, Jin D. Substance $P$ activates the Wnt signal transduction pathway and enhances the differentiation of mouse preosteoblastic MC3T3-E1 cells. Int J Mol Sci. 2014;15:6224-40

44. Guo J, Liu M, Yang D, Bouxsein ML, Saito H, Galvin RJ, Kuhstoss SA, Thomas CC, Schipani E, Baron R. Suppression of Wnt signaling by Dkk1 attenuates PTH-mediated stromal cell response and new bone formation. Cell Metab. 2010;11:161-71.

45. Tamura M, Sato MM, Nashimoto M. Regulation of CXCL12 expression by canonical Wnt signaling in bone marrow stromal cells. Int J Biochem Cell Biol. 2011;43:760-7.

46. Georgiou KR, King TJ, Scherer MA, Zhou H, Foster BK, Xian CJ. Attenuated $\mathrm{Wnt} / \beta$-catenin signalling mediates methotrexate chemotherapy-induced bone loss and marrow adiposity in rats. Bone. 2012;50:1223-33.

47. Maser RE, Lenhard MJ, Pohlig RT, Balagopale PB. Osteopontin and osteoprotegerin levels in type 2 diabetes and their association with cardiovascular autonomic function. J Diabetes Complicat. 2016;30:507-10.

48. Chen W, ten Berge D, Brown J, Ahn S, Hu LA, Miller WE, Caron MG, Barak LS, Nusse R, Lefkowitz RJ. Dishevelled 2 recruits beta-arrestin 2 to mediate Wnt5A-stimulated endocytosis of frizzled 4. Science. 2003;301:1391-4.

49. Lee YN, Gao Y, Wang HY. Differential mediation of the Wnt canonical pathway by mammalian dishevelleds-1, -2, and -3. Cell Signal. 2008;20:443-52.

50. Zhang LH, Luan L, Ma YY. Dishevelled-2 modulates osteogenic differentiation of human synovial fibroblasts in osteoarthritis. Mol Med Rep. 2018;18:292-8.

51. Gao C, Chen YG. Dishevelled: the hub of Wnt signaling. Cell Signal. 2010;22:717-27.

52. Kafka A, Bašić-Kinda S, Pećina-Šlaus N. The cellular story of dishevelleds. Croat Med J. 2014;55:459-67.

53. Long HT, Sun BH, Cheng L, Zhao SS, Zhu Y, Zhao RB, Zhu JX. miR-139-5p represses BMSC osteogenesis via targeting Wnt/b-catenin signaling pathway. DNA Cell Biol. 2017;36:715-24.

54. Fu HD, Wang BK, Wan ZQ, Lin H, Chang ML, Han GL. Wnt5a mediated canonical Wnt signaling pathway activation in orthodontic tooth movement: possible role in the tension forceinduced bone formation. J Mol Histol. 2016:47:455-66.

\footnotetext{
Ready to submit your research? Choose BMC and benefit from:

- fast, convenient online submission

- thorough peer review by experienced researchers in your field

- rapid publication on acceptance

- support for research data, including large and complex data types

- gold Open Access which fosters wider collaboration and increased citations

- maximum visibility for your research: over $100 \mathrm{M}$ website views per year
}

At BMC, research is always in progress.

Learn more biomedcentral.com/submissions 